\title{
Transient covert attention does alter appearance: A reply to Schneider (2006)
}

\author{
SAM Ling ANd MARisa Carrasco \\ New York University, New York, New York
}

\begin{abstract}
We recently demonstrated that transient covert attention increases the apparent contrast of a stimulus (Carrasco, Ling, \& Read, 2004). Schneider (2006) proposes that the observed increase in apparent contrast is largely due to sensory interactions occurring between the precue and stimulus, rather than to attention. Specifically, he reports that cuing effects only occur at contrasts near detection threshold, and that there are confounding sensory interactions between the cue and stimulus at suprathreshold detection contrasts. Our response is twofold. First, we outline the key methodological differences between our original study and Schneider's that are likely to account for the different results, and explain how we had ruled out the sensory interaction explanation of the cue. Second, we directly test the prediction put forth by Schneider: If the effects were due to sensory interactions, reversing the luminance polarity of the precue in our paradigm should lead to differential cuing effects. We replicate one of the experiments of our original study and add a condition in which the cue luminance is either black or white. Our results replicated our previous findings - they showed an increase in apparent contrast to a high-contrast stimulus when it was precued. Moreover, we found that the black cue and the white cue had the same effect, thus ruling out the alternative explanation proposed by Schneider. Transient attention does alter contrast appearance.
\end{abstract}

Attention is the mechanism that allows us to selectively process the vast amount of information that we receive, and to guide our behavior. Visual spatial attention can be deployed overtly, accompanied by eye movements to the relevant location, or covertly, without such eye movements (Posner, 1980). There are two types of covert attention: sustained and transient (Cheal \& Lyon, 1991; Corbetta \& Shulman, 2002; Nakayama \& Mackeben, 1989). Sustained attention refers to the voluntary, endogenous directing of attention to a location in the visual field. Transient attention refers to the automatic, exogenous grabbing of attention to a location in the visual field, brought about by a transient flash or abrupt onset.

It is well established that attention improves performance - accuracy and speed —on many tasks. However, an important issue debated for over a century by psychologists, philosophers, and physiologists alike is whether attention alters our subjective experience of the visual world. Surprisingly, until recently few studies have directly investigated this critical issue. We will not revisit the specifics here, given that they have already been reported in numerous articles (Carrasco, Ling, \& Read, 2004; Fuller \& Carrasco, 2006; Gobell \& Carrasco, 2005; Hsieh, Caplovitz, \& Tse, 2005; Liu, Fuller, \& Carrasco, 2006; Montagna \& Carrasco, 2006; Prinzmetal, Amiri, Allen, \& Edwards, 1998; Prinzmetal, Nwachuku, Bodanski, Blumenfeld, \& Shimizu, 1997; Schneider, 2006; Tsal \& Shalev, 1996; Tsal, Shalev, Zakay, \& Lubow, 1994; Turatto, Viscovi, \& Valsecchi, 2007).
A critical obstacle in addressing this issue had been the lack of an effective method to measure subjective experience. It is important to distinguish a change in appearance from a response bias that may arise from task demands. Recently, we implemented a paradigm to study the effect of transient covert attention on appearance, which quantifies the observer's subjective perception using a task contingent upon a comparative judgment between two stimuli on a particular feature (Carrasco, Ling, \& Read, 2004). In this paradigm, observers are shown two stimuli and are asked to report property $x$ of the stimulus that is greater/ lesser in property $y$; that is, the perceived relative values of property of $y$ - the primary focus of the experiment (i.e., contrast) - is an indicator of which stimulus to report on property $x$ (i.e., orientation). The critical manipulation is that observers are not asked to directly rate their subjective experience on property $y$ but to make a decision about another stimulus, property $x$.

For instance, in Carrasco, Ling, and Read (2004), we presented two Gabor patches, one to the left and the other to the right of fixation, and asked observers to report the orientation of the stimulus that was higher in contrast. These instructions emphasized the orientation judgment, whereas in fact we were interested in their contrast judgments. On each trial, the "standard" Gabor was of a fixed contrast, but the contrast of the "test" Gabor was randomly chosen from a range of contrasts sampled around the contrast of the standard Gabor. The orientation of each Gabor was chosen randomly. We manipulated transient attention

M. Carrasco, marisa.carrasco@nyu.edu 
with an uninformative peripheral cue, which automatically captured attention to the cued location. The results showed that transient attention significantly increased perceived contrast. This procedure, in conjunction with the control experiments we conducted (see Discussion), rules out response bias (Luck, 2004; Treue, 2004).

Using this paradigm, it has been reported that attention alters our subjective impression of many dimensions: contrast appearance (Carrasco, Ling, \& Read, 2004; Hsieh et al., 2005), spatial frequency and gap size (Gobell \& Carrasco, 2005), flicker rate (Montagna \& Carrasco, 2006), motion coherence (Liu et al., 2006), size of a motion stimulus (Anton-Erxleben, Henrich, \& Treue, 2007), perceived speed (Turatto et al., 2007), and color saturation (but not hue; Fuller \& Carrasco, 2006). For a review, see Carrasco (2007).

Recently, Schneider (2006) has questioned whether attention alters contrast appearance. The crux of his article is that the luminance polarity of the cue (black or white rings surrounding the upcoming stimulus location) affects appearance of brightness contrast differentially, depending on the cue luminance and stimulus contrast. Specifically, Schneider reported that an effect was found on apparent brightness contrast with all cue types tested when the Weber contrast of the stimuli was near detection threshold ( $1 \%-2 \%$ contrast). However, whereas the white peripheral cue increased perceived contrast for suprathreshold targets ( $\sim 3 \%-6 \%$ contrast), black peripheral cues tended to reduce perceived contrast. In addition, a central gaze cue (e.g., Driver, Davis, \& Ricciardelli, 1999) was introduced as a reflexive cue far enough from the stimulus to ensure no sensory interactions. With this gaze cue, Schneider reported a small effect near threshold, and no effect at higher contrasts. On the basis of these results, he suggests that the results of our previous study (Carrasco, Ling, \& Read, 2004) are due to sensory characteristics of the cue and not to the effects of attention.

Our reply is twofold. First, given that Schneider's (2006) study was not a replication of the parameters of the Carrasco, Ling, and Read (2004) study, we indicate the key methodological differences between Schneider's study and ours, which may account for the different results. Second, we present experimental data testing Schneider's central prediction. Faithful to his hypothesis that the effect we reported was due to a sensory interaction, he states "a white cue would be predicted to decrease the perceived contrast of Gabor targets" (p. 809). We therefore directly tested this prediction by using the cue luminance polarities used by Schneider with our paradigm, and showed that this cue luminance polarity manipulation had no differential effect whatsoever on contrast appearance, thereby ruling out a sensory explanation.

\section{Key Methodological Differences Between Schneider (2006) and Carrasco, Ling, and Read (2004)}

Judgments of brightness versus judgments of contrast. We measured the effect of attention on sinusoidally modulated gratings and gathered information regarding perceived Michelson contrast. We made absolutely no claim regarding perceived brightness contrast. Schneider (2006) measured brightness appearance of discs. Although it is possible to convert brightness contrast into Michelson contrast mathematically, perceptually these are very different for the visual system - that is, judging which Gabor appears to be of higher contrast (bright-dark modulation) rather than which dot is brighter. Indeed, it has been shown that luminance and contrast processing are largely independent in natural images, as are the mechanisms of luminance gain control and contrast gain control in the early visual system (Mante, Frazor, Bonin, Geisler, \& Carandini, 2005).

Spatial characteristics of the peripheral attentional cue. The peripheral cue in Schneider's (2006) study differed significantly from ours. We used a relatively small dot $\left(0.1^{\circ}\right)$ appearing $1.5^{\circ}$ above one of the two stimuli, a cue known to direct transient attention to target locations and to affect contrast sensitivity (Carrasco, PenpeciTalgar, \& Eckstein, 2000; Ling \& Carrasco, 2006; Lu \& Dosher, 1998, 2000; Pestilli \& Carrasco, 2005; Solomon, 2004), and spatial resolution (Carrasco, Loula, \& Ho, 2006; Carrasco, Williams, \& Yeshurun, 2002; Yeshurun \& Carrasco, 1998, 1999, 2000), and to accelerate information accrual (Carrasco, Giordano, \& McElree, 2004; Carrasco \& McElree, 2001).

We have conducted a number of experiments to rule out a sensory explanation of the findings we have attributed to transient attention. For instance, in a study investigating the effects of attention on appearance of spatial frequency, a black postcue replaced the black precue. The spatial and temporal contiguity between the cue and stimulus were the same, but whereas the precue increased perceived spatial frequency, the postcue did not alter appearance (Gobell \& Carrasco, 2005).

This result is consistent with an fMRI study investigating the neural basis of transient attention (Liu, Pestilli, \& Carrasco, 2005). They found that precuing the target location increased stimulus-evoked response in corresponding retinotopic striate and extrastriate areas, compared with the control conditions. Liu et al. (2005) ruled out possible sensory contamination brought about by the cue by introducing a spatial and temporal manipulation that complemented each other. First, in the spatial manipulation, by having the cue and stimulus activity isolated to the ventral and dorsal partition of the visual cortex, respectively, they ensured that the response of the cue and stimulus were anatomically separate. In the temporal manipulation, they took advantage of the sluggishness of the hemodynamic response and evaluated the effect of postcue trials to control for the sensory effect of the cue. Because of the sluggishness of the hemodynamic response, a sensory response from a region that responded to both the cue and stimulus could not differentiate the order of the two. These spatial and temporal parameters ruled out a sensory explanation of the enhanced stimulus-evoked response brought about by transient attention (Liu et al., 2005).

Schneider (2006) used three types of cues: white or black abrupt onsets and a central-gaze direction cue located remotely from the targets. The black and white cues were rings surrounding the upcoming stimulus location. The use of this particular type of cue has been shown to create sensory interactions due to paracontrast masking (Averbach \& Coriell, 
1961; Breitmeyer et al., 2006; Ogmen, Breitmeyer, \& Melvin, 2003). It has been suggested that some researchers may have not found attentional effects because they have used suboptimal cues that overlap spatially or cause paracontrast masking with the target (e.g., Luck, Hillyard, Mouloua, \& Hawkins, 1996; Yeshurun \& Carrasco, 1999). Indeed, it is possible that the slight facilitation that Schneider finds with the white ring cue over the black cue may be due to sensory interactions such as paracontrast masking. Paracontrast masking has differential effects on perceived brightness, depending on the polarity of the mask (Breitmeyer et al., 2006). Using rings surrounding an upcoming stimulus location as paracontrast masks and varying the luminance of the ring, it has been shown that at a stimulus onset asynchrony (SOA) of $\sim 120 \mathrm{msec}$ (corresponding to that used by Schneider), apparent brightness contrast is facilitated when the preceding ring is white, whereas there is little to no effect when the ring is black. Although the contrasts of stimuli in that study are higher than those in Schneider's, the trend that he reported for white cues to be more effective than black is consistent with this finding regarding paracontrast masking. Therefore, Schneider's conclusion that his cuing effect was due to sensory interactions is probably true for the particular cue type he used; it might have served more as a paracontrast mask than as an attentional cue. The size and placement of the cue in our original study (Carrasco, Ling, \& Read, 2004) was specifically chosen to elicit transient attention while avoiding any confounds from masking.

\section{Experiment}

Schneider (2006) maintains that the effects observed in Carrasco, Ling, and Read (2004) are not due to attentional effects on apparent contrast, but rather to sensory interactions between the precue and stimuli. He claims that this is particularly the case with higher contrast stimuli $(\sim 3 \%-6 \%$ contrast $)$. This alternative explanation rests on the prediction that changing the luminance of the cues would lead to differences in cuing effects. Although one of the key differences between his study and ours is that he tested brightness contrast and we tested Gabor contrast, in his discussion Schneider makes a specific prediction regarding a sensory interaction effect with our sinusoidally modulated contrast stimuli. In particular, he concludes that a black cue should lead to an increase in perceived Michelson contrast, whereas a white cue should lead to a decrease in perceived Michelson contrast.

Notwithstanding the key differences between Schneider's (2006) study and ours, in the present experiment we directly tested the hypothesis he put forth. We used an exact replication of Experiment 2 in our original study, which used a wide range of contrast stimuli $(6 \%-69 \% \mathrm{Mi}$ chelson contrast; Carrasco, Ling, \& Read, 2004), except that the precues in the original study were black, whereas in the present study they were either black or white.

\section{METHOD}

\author{
Observers \\ Twelve observers participated in the experiment. All had normal \\ or corrected-to-normal vision.
}

\section{Apparatus}

The stimuli were created on a G4 Power Macintosh using MATLAB and the Psychophysics Toolbox (Brainard, 1997; Pelli, 1997). Observers viewed the stimuli on a gamma-corrected monitor (Pelli \& Zhang, 1991). A video attenuator was used to drive just the green gun of a 21 -in. monitor $(1,024 \times 768 ; 120 \mathrm{~Hz})$, thus providing a larger possible set of distinct luminance levels ( 12.6 bits). Background luminance was set to $9.86 \mathrm{~cd} / \mathrm{m}^{2}$. White corresponded to $39.79 \mathrm{~cd} / \mathrm{m}^{2}$, and black to $0.01 \mathrm{~cd} / \mathrm{m}^{2}$.

\section{Stimuli and Design}

A black square $\left(0.1^{\circ} \times 0.1^{\circ}\right)$ was presented in the center of a uniform gray background, serving as a fixation point. The fixation point was presented in the center of the screen throughout the entire experiment. There were four types of cues: white or black neutral and white or black peripheral. The peripheral cue was a small white or black dot $\left(0.3^{\circ} \times 0.3^{\circ}\right)$ that appeared $1.5^{\circ}$ above the center of the location where one of the two stimuli was about to appear. The neutral cue was a white or black dot presented at fixation. The display consisted of two Gabor patches (4-cpd sinusoidal gratings enveloped by a Gaussian; $2^{\circ} \times 2^{\circ}$ ) presented to the left and right of fixation at $4^{\circ}$ eccentricity along the horizontal meridian. From trial to trial, the stimuli randomly varied (with equal probability) in orientation and contrast. The stimuli were independently tilted $10^{\circ}$ either to the left or to the right.

One of the Gabor patches was always presented at a fixed contrast of $21 \%$ (hereafter referred to as the standard), whereas the contrast of the other Gabor (hereafter referred to as the test) was randomly sampled from a set of Michelson contrasts in 9-log increments, from $6.3 \%$ to $69 \%$.

\section{Procedure}

Each observer participated in a practice block of 75 trials and 5 experimental blocks of 200 trials each. Observers viewed the display binocularly at a distance of $114 \mathrm{~cm}$ from the monitor with their heads stabilized by a chinrest. They were asked to fixate on the fixation point throughout the experiment. As illustrated in Figure 1, in each trial observers were presented with a fixation point for $500 \mathrm{msec}$, after which either a white or black peripheral or neutral cue was briefly flashed $(67 \mathrm{msec})$. Following an interstimulus interval of $53 \mathrm{msec}$, the two Gabor stimuli were presented for $40 \mathrm{msec}$.

Observers performed a $2 \times 2$ alternative forced choice (AFC) task during which they indicated the orientation of the Gabor that appeared higher in contrast. They were then asked, "Is the higher contrast stimulus tilted to the left or the right?" If the stimulus to the left of fixation appeared higher in contrast, observers indicated its orientation by pressing either " $Z$ " (leftward tilt) or " $\mathrm{X}$ " (rightward tilt) with the middle or index finger of the left hand. If the stimulus to the right of fixation appeared higher in contrast, observers indicated its orientation by pressing either "." (leftward tilt) or "/" (rightward tilt) with the middle or index finger of the right hand. Observers were told that the peripheral cue was not informative either in terms of contrast or orientation, and that it had an equal probability of appearing on either the higher or lower contrast stimulus.

The $120-\mathrm{msec}$ interval between the cue and target onset was chosen to maximize the effect of the peripheral cue in automatically eliciting transient attention (e.g., Carrasco, Ling, \& Read, 2004; Cheal \& Lyon, 1991; Nakayama \& Mackeben, 1989). Furthermore, the 160 -msec interval between cue onset and stimulus offset was chosen to preclude eye movements (Mayfrank, Kimmig, \& Fischer, 1987), thus ensuring that the observers performed the task under the conditions of covert attention.

\section{RESULTS}

The results are summarized in the average appearance psychometric functions for black precues and white precues (Figure 2, top panel and bottom panel, respectively). 


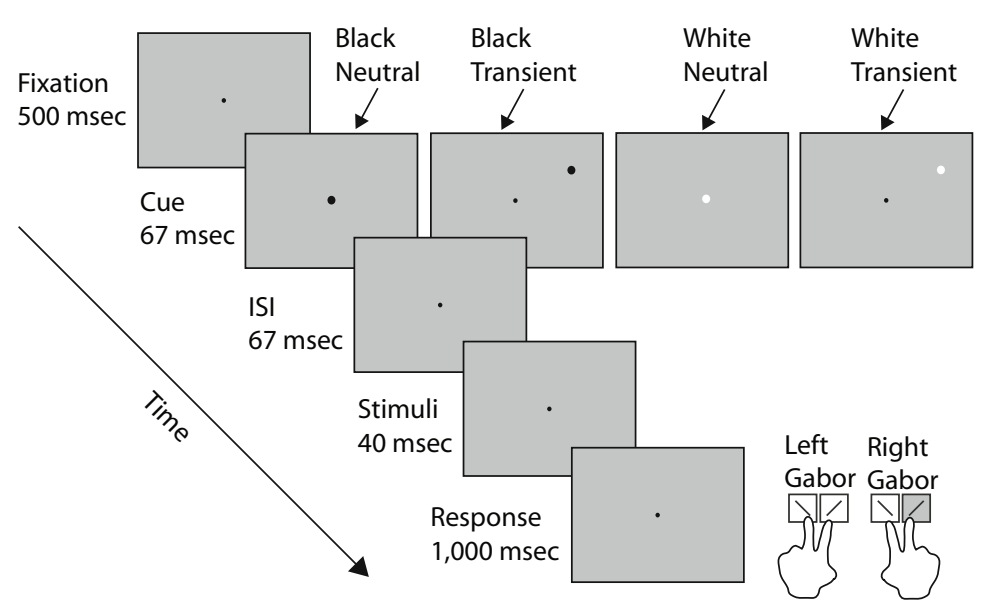

Figure 1. Sequence of events in a single trial. Each trial began with a fixation point followed by a brief neutral or peripheral cue. The luminance of both of these cues could be either white or black. The peripheral cue had equal probability of appearing on the left or right side, and was not predictive of the stimulus contrast or orientation. The timing of this sequence maximized the effect of transient attention and precluded eye movements. Observers performed a $2 \times$ 2 AFC task: They were asked to indicate the orientation (left vs. right) for the higher contrast stimulus.

The data were collapsed across observers and fitted to Weibull functions using maximum likelihood, allowing threshold and slope parameters to vary. The point of subjective equality (PSE) was obtained from these fits as the contrast intensity at which observers' contrast judgments were at chance $(50 \%)$. As expected in the neutral condition, the PSE reflected the veridical percept: When the test and standard were both physically $21 \%$ contrast, observers were at chance of reporting which stimulus appeared higher in contrast. However, when transient attention was drawn to a stimulus via a peripheral cue, the PSE corresponded to lower test contrasts. In other words, cuing the test stimulus reduced the test contrast required to match the standard. Likewise, cuing the standard stimulus increased the test contrast required to match the standard. This was the case for both black and white precues. The results with the black cue replicated what we found in the high contrast condition (Experiment 2) in Carrasco, Ling, and Read (2004). Moreover, the magnitude of attentional effects that we observed in this study is virtually identical to what was found in that study ( $\sim 6 \%$ boost in apparent contrast). Although the high contrasts rendered performance on the orientation discrimination task near asymptote for almost all contrast levels tested, when the test was cued at PSE contrasts, attention improved accuracy from $90 \%$ to $93 \%$ compared with the neutral condition with the black cues, and from $90 \%$ to $94 \%$ with the white cues.

Schneider (2006) predicts that changing the luminance polarity of the peripheral cue should affect the magnitude of the appearance effect. However, in the present experiment, note that this attentional effect occurred whether the cue was black or white, and the magnitude of effects for the two cue luminances was identical. Figure 3 plots the average PSEs for both black and white cues under the test cued, neutral cued, and standard cued conditions. There was no difference between the black and white cues. An ANOVA on PSEs estimated for each observer confirmed these results: There was a main effect of cue type $[F(2,22)=45.81, p<.0001]$. This is a replication of what was found in Carrasco, Ling, and Read (2004): Peripheral cues increase apparent contrast. Critically, we found neither a main effect of cue luminance $[F(1,11)<1]$ nor an interaction of cue type and cue luminance $[F(2,22)<1]$.

The finding that the luminance of the cue made no difference contradicts Schneider's (2006) prediction that a black cue should lead to an increase in perceived Michelson contrast and that a white cue should lead to a decrease in perceived Michelson contrast. Hence, the present experimental findings do not support Schneider's alternative explanation of the cue effect we had found: that it was due to sensory interactions between cue and target.

\section{DISCUSSION}

How attention affects awareness and subjective experience has been approached from various perspectives, most of which are the result of inferences made on the basis of performance- or physiology-based measures, rather than from directly probing observers' subjective experience (e.g., Mack \& Rock, 1998; Simons \& Ambinder, 2005; Tong, 2003). We have implemented a paradigm to probe how transient attention affects observers' subjective experience.

Schneider (2006) proposed that the findings of Carrasco, Ling, and Read (2004) are not due to attentionincreasing apparent contrast, but rather largely due to sensory interactions between the cue and stimuli, particularly at higher contrasts. Schneider's data show that peripheral precues increase perceived brightness contrast only at levels near detection threshold, and the effect 


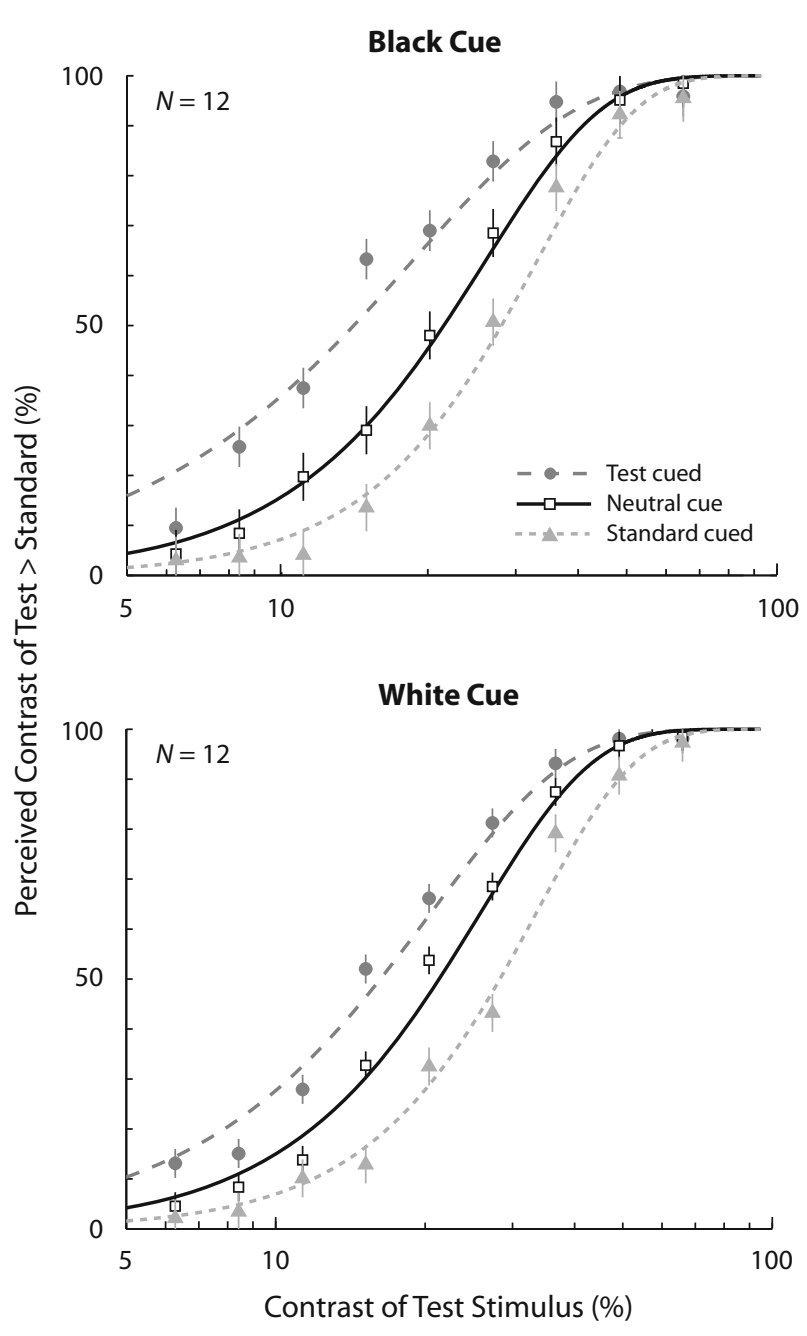

Figure 2. Appearance psychometric functions-proportion of responses in which observers reported the contrast of the test patch as higher than the standard as a function of the test patch's physical contrast - for the neutral and peripheral conditions (test cued and standard cued). The top panel corresponds to psychometric functions when the cue was black, and the bottom corresponds to when the cue was white. In both cases, precuing the test stimulus decreased the contrast of the test needed to perceptually match the standard. Similarly, precuing the standard increased the contrast of the test needed to match the standard. The standard was $21 \%$ contrast. Error bars correspond to $\pm 1 \mathrm{SE}$.

goes away at higher contrasts. He finds that the effect near threshold is greater for white cues than for black cues. It is possible that attention exerts a different effect on perceived brightness (as investigated by Schneider) than on perceived contrast (as investigated in the present study and by Carrasco, Ling, \& Read, 2004). However, this conclusion is limited by the fact that the peripheral cue used in Schneider's study has been shown to also act as a forward, paracontrast mask (Breitmeyer et al., 2006; Ogmen et al., 2003), which could arrest any attentional effects on perceived brightness. Thus, because the precue could have served double duty, both as an attentional cue and as an unintended paracontrast mask, it is possible that Schneider's results are indeed due to sensory contamina- tion of the stimulus by the cue used in that study. Indeed, it has been shown that masks with characteristics similar to Schneider's precue can lead to similar differential effects depending on the luminance of the mask, with white rings increasing apparent brightness more than black rings (Breitmeyer et al., 2006).

We tested Schneider's (2006) prediction-which arises from his proposal that the cue effect found by Carrasco, Ling, and Read (2004) was due to sensory contamination - that white and black cues should have differential effects on perceived Michelson contrast. We replicated the parameters of Experiment 2 of our original study, which used a wide range of contrast stimuli, and we manipulated the luminance polarity of the cue. The present results replicate the finding that peripherally cuing a stimulus increases its apparent contrast, even for highcontrast stimuli. Critically, we found that cue polarity does not matter, indicating that the effect of the cue is not due to sensory factors but rather to transient attention.

Finding the same effect with different cue color is consistent with our previous studies showing similar effects of attention regardless of the type of peripheral cue used. Whereas in many studies investigating the effect of attention on performance in visual tasks a black peripheral cue has been used (Carrasco, Ling, \& Read, 2004; Carrasco et al., 2000; Hsieh et al., 2006; Lu \& Dosher, 1998, 2000), a green cue has shown to affect texture segmentation (Yeshurun \& Carrasco, 1998, 2000) and to improve acuity (Yeshurun \& Carrasco, 1999). Using a black cue, as in the apparent-contrast article referred to (Carrasco, Ling, \& Read, 2004), transient attention has been shown to increase perceived spatial frequency and gap size (Gobell \& Carrasco, 2005), as well as perceived motion coherence (Liu et al., 2006) and perceived speed (Turatto et al., 2007). Using a white precue, we have also reported that transient attention increases perceived flicker (Montagna \& Carrasco, 2006) and perceived color saturation, but not hue (Fuller \& Carrasco, 2006). ${ }^{1}$

\section{Attention and High-Contrast Stimuli}

Schneider (2006) stated that exogenous attentional effects primarily occur near detection threshold. He proposed this as evidence of an additive model of attention, with effects largest at low contrasts ( $1 \%-2 \%$ contrast), and little to no attentional effect for stimuli above $3 \%$ brightness contrast. This finding is inconsistent with the present results, as well as with many previous studies. An additive model, also known as activity gain (Williford \& Maunsell, 2006), predicts a clear qualitative result: The largest effect on perceived contrast at the lowest contrast ranges, which would diminish as a function of increasing contrast. Our present data show a robust effect using high-contrast stimuli (6\%-69\% contrast), which is contrary to what an additive model would predict; rather, our results are more consistent with a multiplicative gain mechanism such as contrast gain. Given the nature of the ring cue used by Schneider, it is possible that the additive effect he reported was a result of paracontrast masking, and not an attentional effect.

Schneider (2006) states that Carrasco, Ling, and Read (2004) and Cameron, Tai, and Carrasco (2002) both ob- 


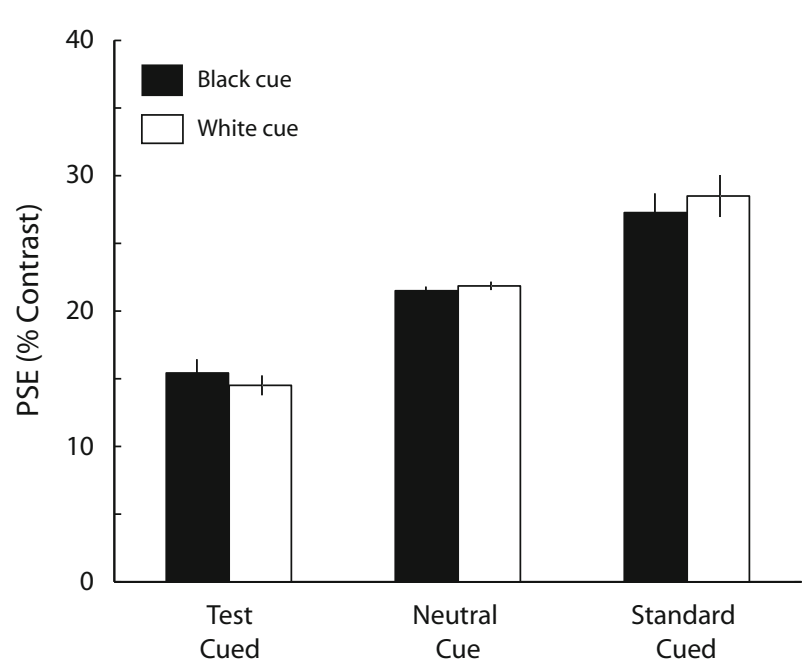

Figure 3. Cue luminance polarity does not affect the effect of attention on apparent contrast. As expected, the average points of subjective equality (PSEs) showed a decrease for the test when the test stimulus was precued, and an increase for the test when the standard was cued. However, there was no significant difference between the black and white cues, indicating that the effect was not due to sensory interactions. Error bars correspond to $\pm 1 \mathrm{SE}$.

serve the greatest attentional effects at low contrasts. However, this is not the case in either study. In Carrasco, Ling, and Read, the effects are quite similar between the low-contrast and high-contrast stimuli (compare results of Figures 2 and 5, and see the Discussion, p. 312, paragraph 2). In addition, Ling and Carrasco (2006) have recently shown that a response gain model best describes the effects of transient attention, whereby attentional effects increase multiplicatively as a function of stimulus contrast. Needless to say, not all attention effects are mediated by enhanced contrast. A number of studies have found attentional effects with contrast stimuli well above detection threshold (Cameron et al., 2002; Carrasco, 2006; Carrasco et al., 2000; Lu \& Dosher, 1998, 2000; Pestilli \& Carrasco, 2005; Solomon, 2004). Moreover, we have been able to show that some attentional effects, such as increased apparent spatial frequency (Gobell \& Carrasco, 2005) or increased perceived flicker rate (Montagna \& Carrasco, 2006), are not mediated by increased apparent contrast.

\section{Direct Versus Indirect Judgments of Appearance}

Another difference between Schneider's (2006) study and our study lies in the way that appearance was measured. We implemented a paradigm that allowed us to measure perceived contrast without asking observers to do so directly. Moreover, because our observers performed an orientation discrimination task contingent upon appearance, we had an objective index indicating that the observers were performing the task and that the cue was effective (Anton-Erxleben et al., 2007; Carrasco, Ling, \& Read, 2004; Fuller \& Carrasco, 2006; Liu et al., 2006). Many have commented on the strengths of this simple paradigm (Luck, 2004; Treue, 2004). In contrast, Schneider asked observers to make ap- pearance judgments directly, and did not gather an objective index indicating that the cue was effective in his appearance studies.

\section{Cue Effectiveness}

When no effect of attention is found on appearance, as was the case when Schneider (2006) used higher contrast, the burden of proof lies in showing that the cue was effective with regard to the standard signature of attention, an improvement of performance at attended locations. For instance, a study revealing that attention increases apparent color saturation but not apparent hue showed that the cue improved performance (orientation discrimination) in both dimensions (Fuller \& Carrasco, 2006). In both the original and present study, we showed that the cue was effective in improving performance as well.

Unfortunately, in Schneider (2006) there is no independent evidence of the efficacy of the ring cue, and only indirect evidence regarding the efficacy of the gaze cue. In Experiment 3, to show that attention was deployed, Schneider reports that a gaze cue speeded response times in a detection task. Given that the crux of the argument rests on differential results with the black and the white cues, and that the results from performance in a speeded detection task with the gaze cue do not necessarily generalize to the appearance results in a discrimination task with a ring cue, it would have been more informative to assess discrimination performance for the two peripheral ring cue conditions instead. Moreover, a speeded response for the gaze cue could be due to a higher asymptote with constant information accrual times (discriminability), to changes in decision criteria, or to accelerated information accrual (Dosher, 1976; McElree \& Carrasco, 1999). Furthermore, several experimental parameters in Schneider's speeded detection task do not correspond to those of his appearance experiments; for instance, the display characteristics did not match (one stimulus rather than two). Thus, little can be concluded from the only experiment conducted to assess cue effectiveness.

\section{Cue Bias}

In our previous appearance studies, observers have been told that the cue appears with equal probability adjacent to either of the two Gabors, so that responding based on the presence of the cue could not be a helpful strategy either to select the higher contrast stimulus (or any other dimension we have tested) or discriminate its orientation. Notwithstanding these methodological precautions, in previous studies we have conducted several control studies to rule out a cue bias explanation of our appearance results. For instance, in some control experiments, we have reversed the direction of the question: We have asked observers to report the orientation of the Gabor of lower, rather than higher, apparent contrast (Carrasco, Ling, \& Read, 2004), spatial frequency (Gobell \& Carrasco, 2005), color saturation (Fuller \& Carrasco, 2006), or flicker rate (Montagna \& Carrasco, 2006). In the contrast study, results indicated that transient attention increased perceived contrast. When asked to indicate the Gabor of lower contrast, observers chose the cued test stimulus less frequently (Carrasco, 
Ling, \& Read, 2004, Experiment 3, Figure 6). Had results been due to cue bias, observers would have chosen the cued stimulus regardless of the direction of the question.

In other control experiments, we have extended the interval between the cue and target onset to $500 \mathrm{msec}-$ timed such that transient attention is no longer active. Transient attention peaks at $\sim 120 \mathrm{msec}$ and decays by $\sim 250 \mathrm{msec}$ (Nakayama \& Mackeben, 1989). Thus, a long interval between the cue and target should eliminate any effect that it may have on perception, and any residual difference between the neutral and peripheral cues would be attributed to a cue bias. When the cue preceded the display by $500 \mathrm{msec}$, there were no systematic differences between the neutral and peripheral conditions in the appearance of contrast (Carrasco, Ling, \& Read, 2004) and of motion coherence (Liu et al., 2006). Thus, when transient attention was no longer active, the appearance of the stimulus was not altered.

\section{Conclusion}

We have presented and discussed evidence indicating that the effect of peripheral cueing on apparent contrast in Carrasco, Ling, and Read (2004) is not due to alternative explanations such as sensory contamination or cue bias, but rather due solely to transient attention; attention increases apparent contrast. This conclusion is consistent with the "linking hypothesis," which states that the attentional enhancement of neural firing is interpreted as if the stimulus had a higher contrast (Reynolds \& Chelazzi, 2004; Treue, 2004). This proposal is supported by converging evidence from neurophysiological (Martinez-Trujillo \& Treue, 2002, Reynolds, Pasternak, \& Desimone, 2000), psychophysical (e.g., Carrasco, Ling, \& Read, 2004; Carrasco, Penpeci-Talgar, \& Eckstein, 2000; Lu \& Dosher, 2000) and neuroimaging (Liu, Pestilli, \& Carrasco, 2005) studies (for a review, see Carrasco, 2006).

\section{AUTHOR NOTE}

The authors thank Stuart Fuller and Taosheng Liu, as well as the other members of the Carrasco lab for their valuable comments and suggestions. This work was funded by National Research Service Award Grant 5 F31 NS051111-03 to S.L. and National Institutes of Health Grant R01 EY016200-01A2 to M.C. Correspondence concerning this article should be addressed to M. Carrasco, Department of Psychology, New York University, New York, NY 10003 (e-mail: marisa .carrasco@nyu.edu).

\section{REFERENCES}

Anton-Erxleben, K., Henrich, C., \& Treue, S. (2007). Attention changes perceived size of moving visual patterns. Journal of Vision, 7 (11), 1-9.

Averbach, E., \& Coriell, A. S. (1961). Short-term memory in vision. Bell Systems Technical Journal, 40, 309-328.

Brainard, D. H. (1997). The Psychophysics Toolbox. Spatial Vision, 10, 433-436.

Breitmeyer, B. G., Kafaligonul, H., Ogmen, H., Mardon, L., ToDD, S., \& ZIEGLER, R. (2006). Meta- and paracontrast reveal differences between contour and brightness processing mechanisms. Vision Research, 46, 2645-2658.

Cameron, E. L., Tai, J. C., \& Carrasco, M. (2002). Covert attention affects the psychometric function of contrast sensitivity. Vision Research, 42, 949-967.
Carrasco, M. (2006). Covert attention increases contrast sensitivity: Psychophysical, neurophysiological and neuroimaging studies. Progress in Brain Research, 154, 33-70.

CARrasco, M. (2007). Visual attention alters appearance: Psychophysical studies of subjective experience. In P. Wilken, T. Bayne, \& A. Cleeremans (Eds.), Oxford companion to consciousness. Oxford: Oxford University Press.

Carrasco, M., Giordano, A. M., \& McElree, B. (2004). Temporal performance fields: Visual and attentional factors. Vision Research, 44, 1351-1365.

Carrasco, M., Ling, S., \& Read, S. (2004). Attention alters appearance. Nature Neuroscience, 7, 308-313.

Carrasco, M., Loula, F., \& Ho, Y.-X. (2006). How attention enhances spatial resolution: Evidence from selective adaptation to spatial frequency. Perception \& Psychophysics, 68, 1004-1012.

Carrasco, M., \& McElree, B. (2001). Covert attention accelerates the rate of visual information processing. Proceedings of the National Academy of Sciences, 98, 5363-5367.

Carrasco, M., Penpeci-Talgar, C., \& Eckstein, M. (2000). Spatial covert attention increases contrast sensitivity across the CSF: Support for signal enhancement. Vision Research, 40, 1203-1215.

Carrasco, M., Williams, P. E., \& Yeshurun, Y. (2002). Covert attention increases spatial resolution with or without masks: Support for signal enhancement. Journal of Vision, 2, 467-479.

Cheal, M., \& Lyon, D. R. (1991). Central and peripheral precuing of forced-choice discrimination. Quarterly Journal of Experimental Psychology, 43A, 859-880.

Corbetta, M., \& Shulman, G. L. (2002). Control of goal-directed and stimulus-driven attention in the brain. Nature Reviews Neuroscience, 3, 201-215.

Dosher, B. A. (1976). The retrieval of sentences from memory: A speed-accuracy study. Cognitive Psychology, 8, 291-310.

Driver, J., Davis, G., \& Ricciardelli, P. (1999). Gaze perception triggers reflexive visuospatial orienting. Visual Cognition, 6, 509-540.

Fuller, S., \& Carrasco, M. (2006). Exogenous attention and color perception: Performance and appearance of saturation and hue. Vision Research, 46, 4032-4047.

Gobell, J., \& CARRASCO, M. (2005). Attention alters the appearance of spatial frequency and gap size. Psychological Science, 16, 644-651.

Hsieh, P. J., Caplovitz, G. P., \& Tse, P. U. (2005). Illusory rebound motion and the motion continuity heuristic. Vision Research, $\mathbf{4 5}$, 2972-2985.

Ling, S., \& CARrasco, M. (2006). Sustained and transient covert attention enhance the signal via different contrast response functions. Vision Research, 46, 1210-1220.

LiU, T., Fuller, S., \& Carrasco, M. (2006). Attention alters the appearance of motion coherence. Psychonomic Bulletin \& Review, 13, 1091-1096.

Liu, T., Pestilli, F., \& Carrasco, M. (2005). Transient attention enhances perceptual performance and FMRI response in human visual cortex. Neuron, 45, 469-477.

Lu, Z. L., \& Dosher, B. A. (1998). External noise distinguishes mechanisms of attention. Vision Research, 38, 1183-1198.

Lu, Z. L., \& Dosher, B. A. (2000). Spatial attention: Different mechanisms for central and peripheral temporal precues? Journal of Experimental Psychology: Human Perception \& Performance, 26, 15341548.

LuCK, S. J. (2004). Understanding awareness: One step closer. Nature Neuroscience, 7, 208-209.

Luck, S. J., Hillyard, S. A., Mouloua, M., \& Hawkins, H. L. (1996). Mechanisms of visual-spatial attention: Resource allocation or uncertainty reduction? Journal of Experimental Psychology: Human Perception \& Performance, 22, 725-737.

MAcK, A., \& Rock, I. (1998). Inattentional blindness. Cambridge, MA: MIT Press.

Mante, V., Frazor, R. A., Bonin, V., Geisler, W. S., \& Carandini, M. (2005). Independence of luminance and contrast in natural scenes and in the early visual system. Nature Neuroscience, 8, 1690-1697.

Martinez-Trujillo, J., \& Treue, S. (2002). Attentional modulation strength in cortical area MT depends on stimulus contrast. Neuron, 35, 365-370.

Mayfrank, L., Kimmig, H., \& Fischer, B. (1987). The role of attention 
in the preparation of visually guided saccadic eye movements in man. In J. K. O'Regan \& A. Levy-Schoen (Eds.), Eye movements: From physiology to cognition. New York: Elsevier, North-Holland.

McElree, B., \& Carrasco, M. (1999). Temporal dynamics of visual search: A speed-accuracy analysis of feature and conjunction searches. Journal of Experimental Psychology: Human Perception \& Performance, 25, 1517-1539.

Montagna, B., \& Carrasco, M. (2006). Transient covert attention and the perceived rate of flicker. Journal of Vision, 6, 955-965.

NaKaYAma, K., \& Mackeben, M. (1989). Sustained and transient components of focal visual attention. Vision Research, 29, 1631-1647.

Ogmen, H., Breitmeyer, B. G., \& Melvin, R. (2003). The what and where in visual masking. Vision Research, 43, 1337-1350.

Pelli, D. G. (1997). The VideoToolbox software for visual psychophysics: Transforming numbers into movies. Spatial Vision, 10, 437-442.

Pelli, D. G., \& Zhang, L. (1991). Accurate control of contrast on microcomputer displays. Vision Research, 31, 1337-1350.

Pestilli, F., \& CARRASCO, M. (2005). Attention enhances contrast sensitivity at cued and impairs it at uncued locations. Vision Research, 45, 1867-1875.

PosNer, M. I. (1980). Orienting of attention. Quarterly Journal of Experimental Psychology, 32, 3-25.

Prinzmetal, W., Amiri, H., Allen, K., \& Edwards, T. (1998). Phenomenology of attention: 1 . Color, location, orientation, and spatial frequency. Journal of Experimental Psychology: Human Perception \& Performance, 24, 261-282.

Prinzmetal, W., Nwachuku, I., Bodanski, L., Blumenfeld, L., \& Shimizu, N. (1997). The phenomenology of attention: 2. Brightness and contrast. Consciousness \& Cognition, 6, 372-412.

Reynolds, J. H., \& Chelazzi, L. (2004). Attentional modulation of visual processing. Annual Review of Neuroscience, 27, 611-647.

Reynolds, J. H., Pasternak, T., \& Desimone, R. (2000). Attention increases sensitivity of V4 neurons. Neuron, 26, 703-714.

SCHNEIDER, K. A. (2006). Does attention alter appearance? Perception \& Psychophysics, 68, 800-814.

Simons, D. J., \& AMbinder, M. S. (2005). Change blindness: Theory and consequence. Current Directions in Psychological Science, 14, $44-48$.

Solomon, J. A. (2004). The effect of spatial cues on visual sensitivity. Vision Research, 44, 1209-1216.

Tong, F. (2003). Primary visual cortex and visual awareness. Nature Reviews Neuroscience, 4, 219-229.

Treue, S. (2004). Perceptual enhancement of contrast by attention. Trends in Cognitive Sciences, 8, 435-437.

Tsal, Y., \& SHALEV, L. (1996). Inattention magnifies perceived length: The attentional receptive field hypothesis. Journal of Experimental Psychology: Human Perception \& Performance, 22, 233-243.

Tsal, Y., Shalev, L., ZaKaY, D., \& Lubow, R. E. (1994). Attention reduces perceived brightness contrast. Quarterly Journal of Experimental Psychology, 47A, 865-893.

Turatto, M., Viscovi, M., \& Valsecchi, M. (2007). Attention makes moving objects be perceived to move faster. Vision Research, 47, 166178

Williford, T., \& Maunsell, J. H. (2006). Effects of spatial attention on contrast response functions in macaque area V4. Journal of Neurophysiology, 96, 40-54.

YeSHURUN, Y., \& CARRASCO, M. (1998). Attention improves or impairs visual performance by enhancing spatial resolution. Nature, 396, 72-75.

Yeshurun, Y., \& CARRASCO, M. (1999). Spatial attention improves performance in spatial resolution tasks. Vision Research, 39, 293-306.

Yeshurun, Y., \& CARRASCO, M. (2000). The locus of attentional effects in texture segmentation. Nature Neuroscience, 3, 622-627.

\section{NOTE}

1. We have also evaluated whether cue polarity modifies the attention effect on saturation appearance and found that black cues (Fuller \& Carrasco, 2006) and white cues (unpublished data) yield the same increase in apparent saturation.

(Manuscript received November 14, 2006; revision accepted for publication February 15, 2007.) 